\title{
The Effect of Inoperative Readout Layers on SDC Calorimetry
}

\author{
Dan Green \\ Fermi National Accelerator Laboratory \\ P.O. Box 500, Batavia, Illinois 60510
}

August 1993 


\section{Disclaimer}

This report was prepared as an account of work sponsored by an agency of the United States Government. Neither the United States Government nor any agency thereof, nor any of their employees, makes any warranty, express or implied, or assumes any legal liability or responsibility for the accuracy, completeness, or usefulness of any information, apparatus, product, or process disclosed, or represents that its use would not infringe privately owned rights. Reference herein to any specific commercial product, process, or service by trade name, trademark, manufacturer, or otherwise, does not necessarily constitute or imply its endorsement, recommendation, or favoring by the United States Government or any agency thereof. The views and opinions of authors expressed herein do not necessarily state or reflect those of the United States Government or any agency thereof. 


\title{
THE EFFECT OF INOPERATIVE READOUT LAYERS ON SDC CALORIMETRY
}

\author{
Dan Green \\ Fermi National Accelerator Laboratory \\ Batavia, Illinois
}

August 1993

\section{Introduction}

The SDC calorimeter is to be constructed using $\mathrm{Pb}$ and $\mathrm{Fe}$ absorbers and scintillator active sampling using the tile/fiber technique [1]. In this note, the effect of the inoperative readout of a single sampling layer is studied. The goal of this study is to inform on a cost/benefit analysis of the need to repair inoperative layers of the calorimetric readout.

\section{Use of HF data}

The "hanging file" = HF test apparatus is described elsewhere [2]. It is a reasonable approximation to the SDC central calorimeter [1]. In this note, the effect of an inoperative layer on hadronic (HAD) calorimetry is studied. HF data at a nominal energy of $250 \mathrm{GeV}$ (observed mean energy $\langle\mathrm{E}\rangle=272.30 \mathrm{GeV}$ ) were used. The data consist of calibrated readout of each layer of a 95 layer "stack". The stack has 40 plates of $1 / 8$ " $\mathrm{Pb}(22.8 \mathrm{Xo})$ followed by 55 plates of 1 " Fe $(79.2 \mathrm{Xo})$ interspersed with $2.5 \mathrm{~mm}$ plastic scintillator.

The calibration was done using 1 "mip" muons in each compartment separately; the electromagnetic (EM) and hadronic (HAD). There is one overall relative calibration of compartments. It was chosen to make the e/ $\pi$ response roughly energy independent [2], not to minimize the energy resolution. The EM compartment is about $22.8 \mathrm{Xo}$ of $\mathrm{Pb}$ and 0.75 absorbtion lengths. The HAD compartment is 79.2 Xo of absorber or 8.34 absorption lengths. Typical single events due to $250 \mathrm{GeV}$ incident pions are shown in Fig. 1. Clearly, the hadronic energy depositions have large fluctuations. The mean of many single events is shown in Fig.2. The curve of Fig. 2 is the more conventional "mean hadronic shower depth profile". However, reference to Fig. 1 shows that the large event to event fluctuations have been averaged away. 
A previous study [3] came to some preliminary conclusions about the magnitude of the losses due to the existence of inoperative layers.

\section{Effect of an inoperative layer on calorimeter performance}

The HF stack total energy, found by adding all the layers in the stack, is shown in Fig.3 for $250 \mathrm{GeV}$ incident pions. The mean is, $\langle\mathrm{E}\rangle=272.3 \mathrm{GeV}$, while the second moment, the $\mathrm{rms}$ is, $\mathrm{dE}=14.42 \mathrm{GeV}$. The fractional energy error is then, $\mathrm{dE} / \mathrm{E}=0.053$.

It is assumed that the calorimeter as an energy measuring device is defined by the first and second moments of the reported energy distribution. A broken tile is simulated by dropping one of the 95 independent scintillator readouts from the total energy sum.

The effect of this procedure on the mean of the energy is shown in Fig 4. For layers $<40$ (EM compartment) and layers $>70$ (HAD2 compartment) [1], the loss of a layer causes a shift of $<1 \%$ in the energy response. The maximum shift, occuring at the shower maximum as shown in Fig. 2, is 5.5\%. Note that Fig. 2 and Fig. 4 are essentially the same in shape, as they should be. In fact, the peak value/layer is $\sim 12 \mathrm{GeV}$, which contributes $\sim 5 \%$ to the energy sum. Hence, a failure of this layer would cause an average shift of $\sim 5 \%$ in the energy mean. It should be noted that, if the failure of the layer is detected, for example by moving radioactive sources as are planned for the SDC calorimeters [1], then the tower may be recalibrated knowing the location of the failure, using Fig. 4.

The procedure outlined above evades the effect of the broken layer on the mean. However, the fluctuations on showers about that mean, see Fig. 1, cannot be evaded, nor easily corrected for. In Fig. 5 is shown the effect of a broken layer on the rms of the energy distribution. For layers $<40(\mathrm{EM})$ and $>70(\mathrm{HAD} 2)$ there are $<5 \%$ increases in the rms of the energy. However, the region around the EM/HAD boundary is very sensitive. If the dropped tile is in the first few readout layers in the HAD compartment, then the resolution suffers an increase of up to $22 \%$. In contrast, if a layer near the hadronic shower maximum is dropped, the resolution actually improves. 
The explanation for this behavior is that the SDC calorimeter is a composite object. It consists of $\mathrm{Pb}$ and $\mathrm{Fe}$ absorbers. As stated above, the relative calibration of the $\mathrm{EM}$ and HAD compartments was floated such to make the electron/ $\pi$ response near to 1 and nearly energy independent. This choice of calibration does not minimize the energy resolution [2]. Therefore, it is not implausible that the rms might actually be reduced by imposing a different effective calibration by dropping 1 layer from the HAD energy sum. Note also that the character of the electromagnetic component of the hadronic shower changes at the $\mathrm{Pb} / \mathrm{Fe}$ boundary since the relative radiation length/absorption length differs greatly in the 2 materials. The critical energy, which defines the cutoff energy of an electromagnetic shower [4], also differs greatly as it scales roughly as $1 / Z$.

Therefore, the integrity of the readout in a composite calorimeter such as that proposed by $\mathrm{SDC}$ is important at the $\mathrm{Fe} / \mathrm{Pb}$ boundary. It is expected that a homogeneous calorimeter structure would not exhibit the same measure of sensitivity to the boundary between readout compartments.

\section{REFERENCES}

1. SDC Technical Design Report, SDC-92-101 (1992).

2. A. Beretvas, et al., "Beam Tests of Composite Calorimeter Configurations from Reconfigurable-Stack Calorimeter", Nuc. Inst. Meth., A329 (1993) 50-61.

3. D. Green, "Dead Material and Energy Measurements in Hadronic Calorimeters", Fermilab-TM-1824, January 1993.

4. Review of Particle Properties, Phys. Rev. D 45 (1992). 


\section{FIGURE CAPTIONS}

Fig. 1 Depth profile in the HF stack for $250 \mathrm{GeV}$ incident pions. The first 4 events in a file were chosen. The fluctuations in the hadronic shower shapes are quite evident.

Fig. 2 The average depth profile, obtained by summing the individual profiles shown in Fig. 1 for many incident pions. Note that the fluctuations are smoothed out, and the transition from EM to HAD compartment at plate \#40 is evident.

Fig. 3 Energy response of the calorimeter obtained from summing the 95 readout layers of the entire HF stack. The mean is, $\langle\mathrm{E}\rangle=272.30 \mathrm{GeV}$ and the $\mathrm{rms}$ is $\mathrm{dE}=$ $14.42 \mathrm{GeV}$, or $\mathrm{dE} / \mathrm{E}=0.053$.

Fig. 4 Mean energy with 1 inoperative layer at location $=\mathrm{j}$ in the stack, scaled to the mean of the intact stack, $\langle E(j)>/<E>$, as a function of $j$.

Fig. 5 The rms energy with 1 inoperative layer at location $=\mathrm{j}$ in the stack, scaled to the rms of the intact stack, $\mathrm{dE}(\mathrm{j}) / \mathrm{dE}$, as a function of $\mathrm{j}$. 

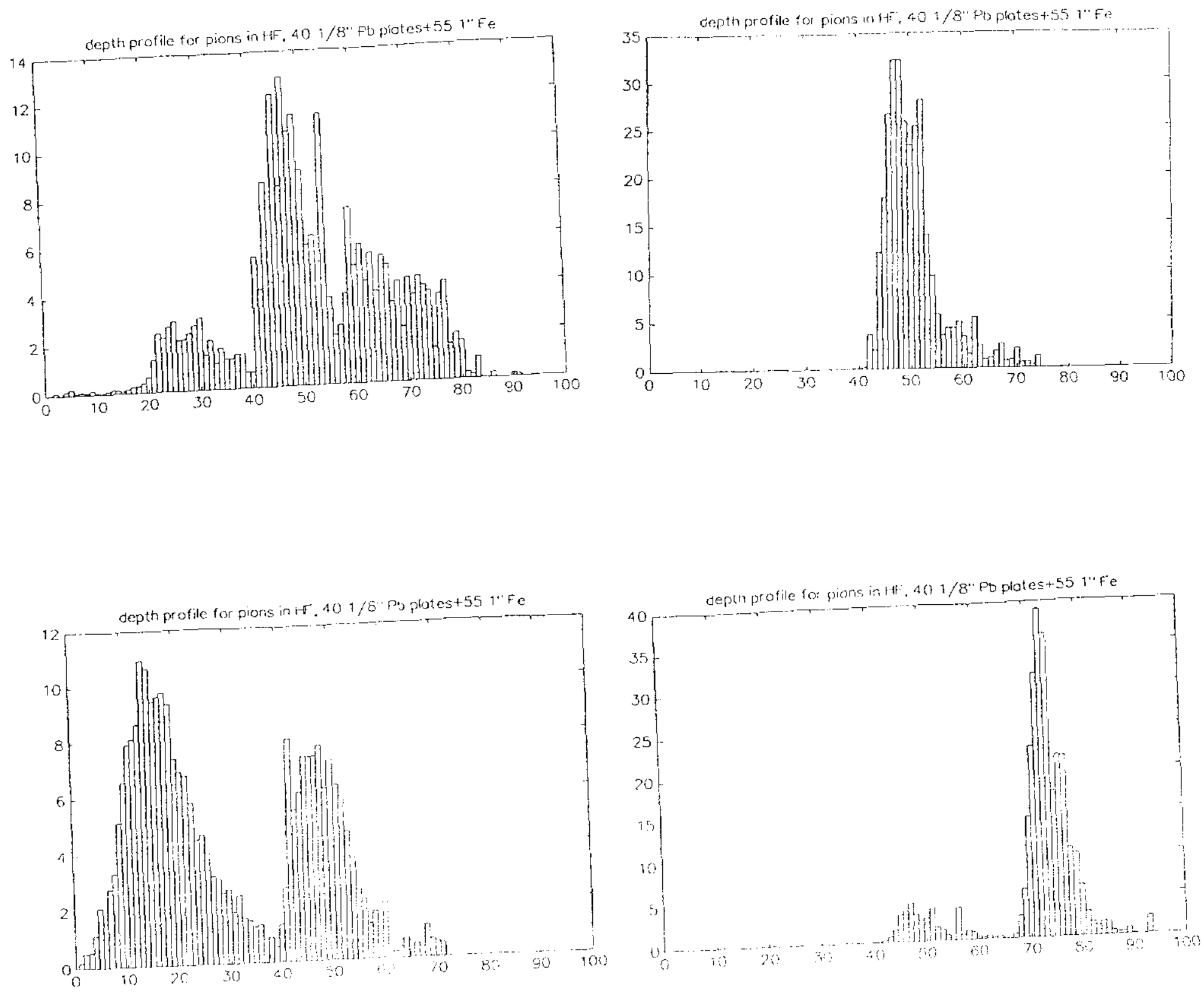

Fig. 1 Depth profile in the HF stack for $250 \mathrm{GeV}$ incident pions. The first 4 events in a file were chosen. The fluctuations in the hadronic shower shapes are quite evident. 


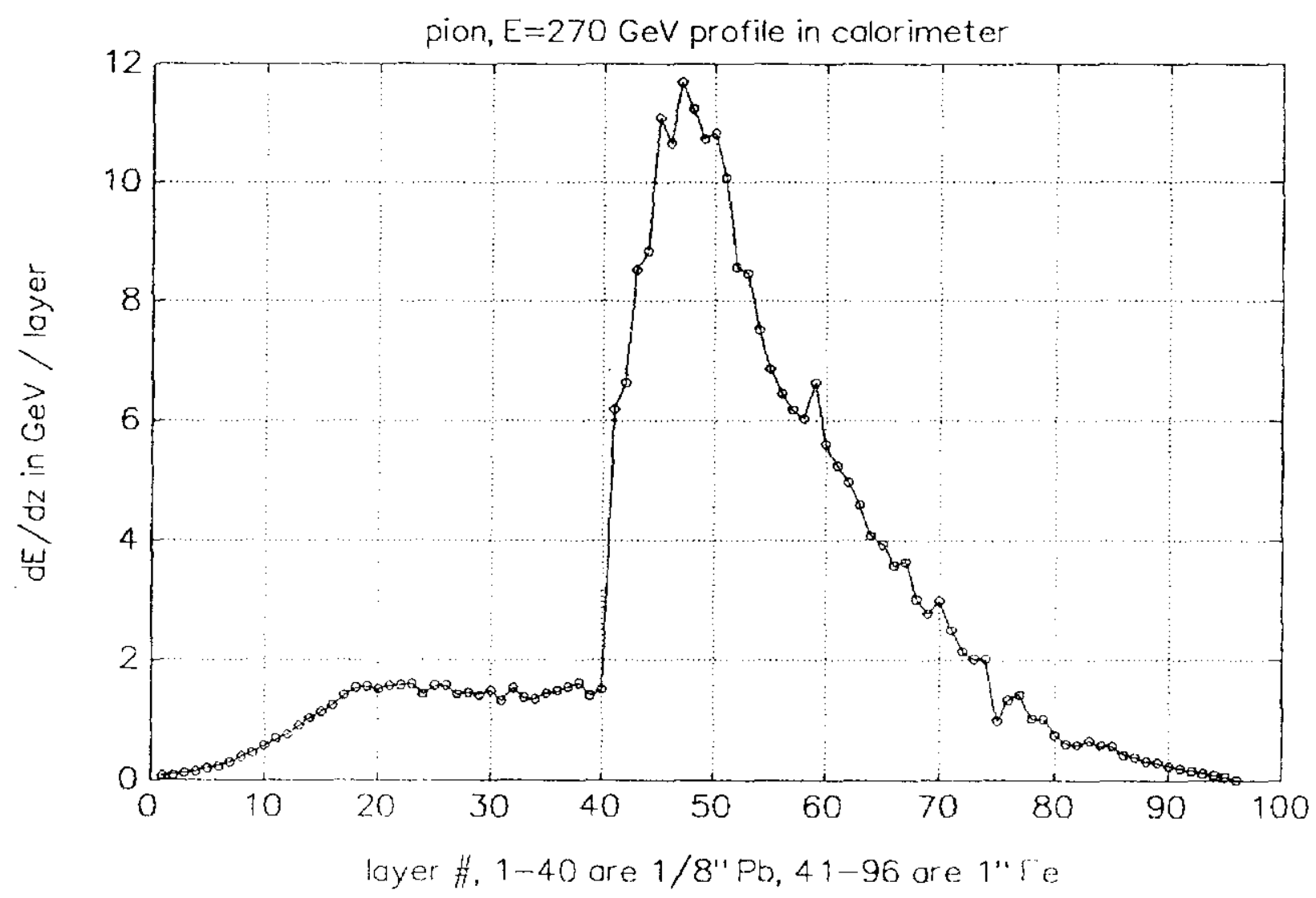

Fig. 2 The average depth profile, obtained by summing the individual profiles shown in Fig. 1 for many incident pions. Note that the fluctuations are smoothed out, and the transition from EM to HAD compartment at plate \#40 is evident. 
HF pion doto, energy response

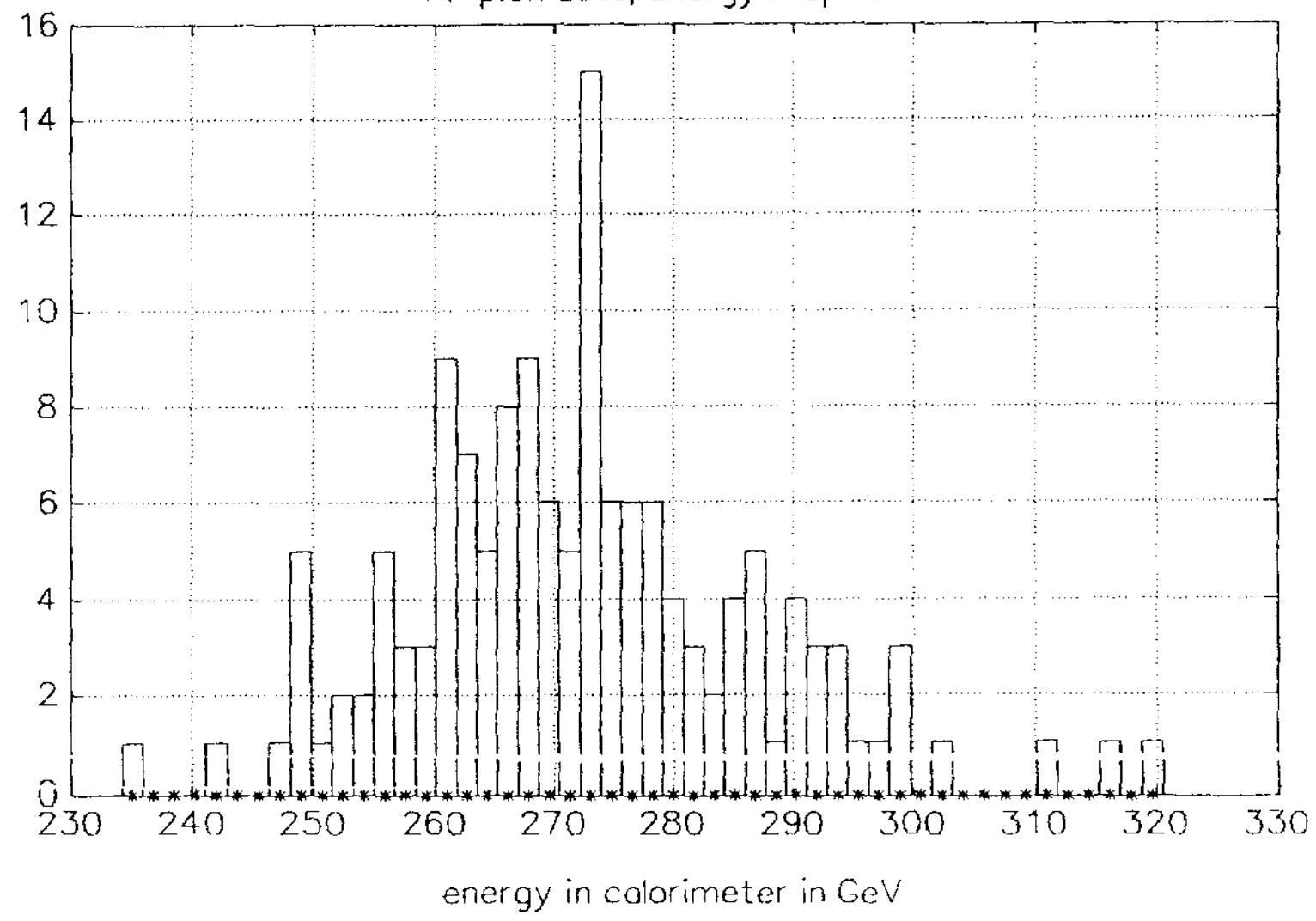

Fig. 3 Energy response of the calorimeter obtained from summing the 95 readout layers of the entire HF stack. The mean is, $\langle\mathrm{E}\rangle=272.30 \mathrm{GeV}$ and the $\mathrm{rms}$ is $\mathrm{dE}=$ $14.42 \mathrm{GeV}$, or $\mathrm{dE} / \mathrm{E}=0.053$. 


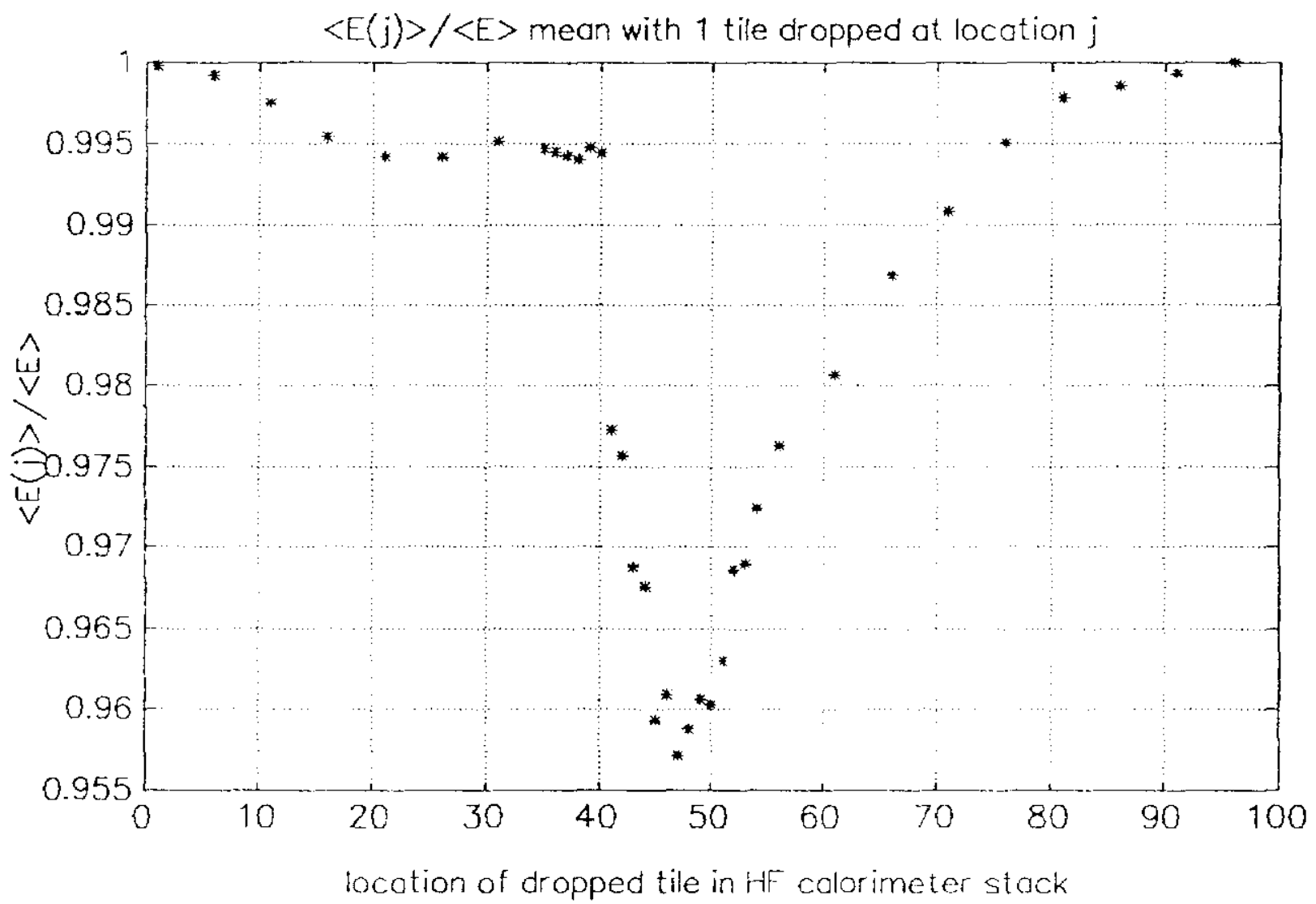

Fig. 4 Mean energy with 1 inoperative layer at location $=\mathrm{j}$ in the stack, scaled to the mean of the intact stack, $\langle E(j)>\mid<E\rangle$, as a function of $j$. 


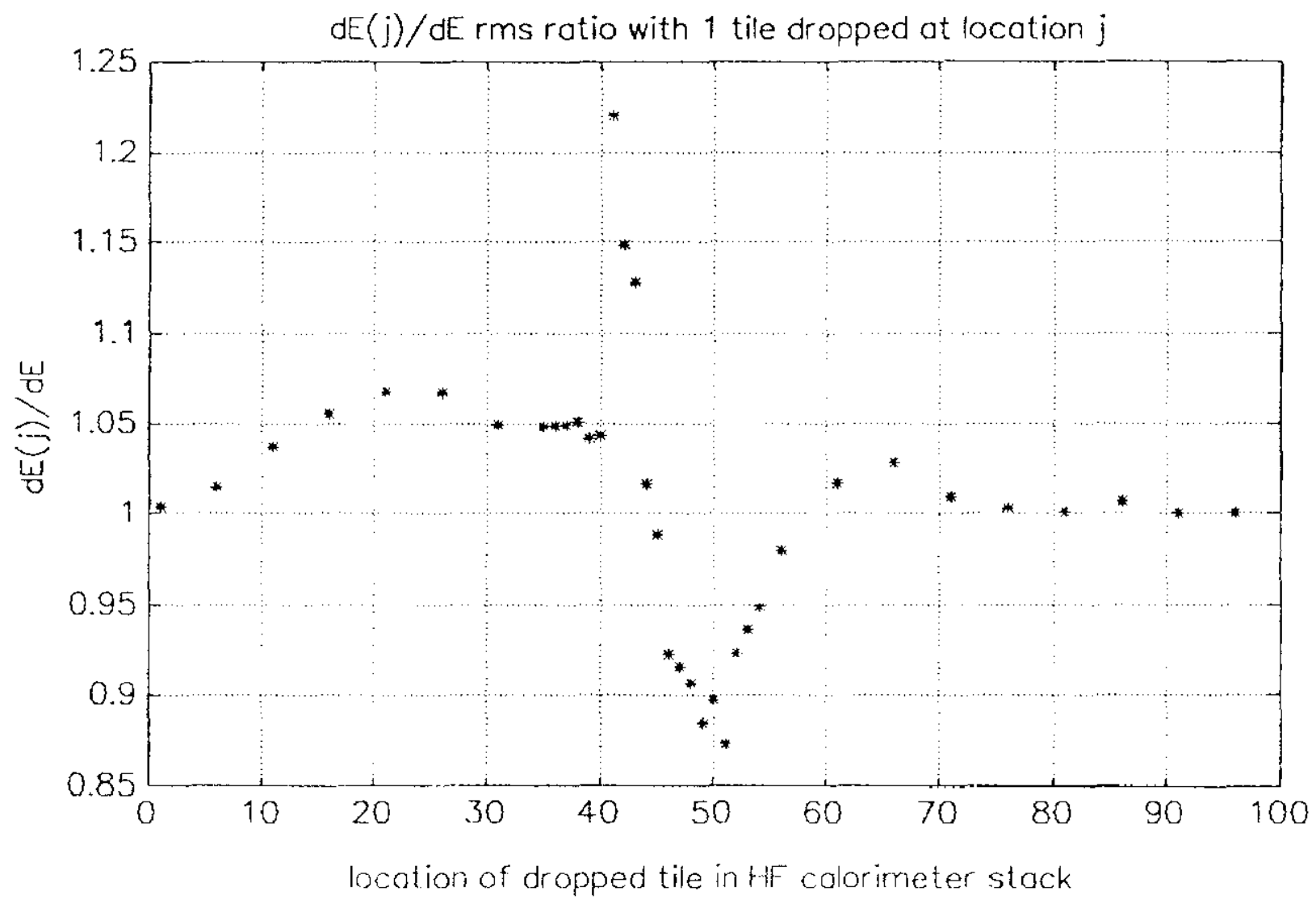

Fig. 5 The rms energy with 1 inoperative layer at location $=\mathrm{j}$ in the stack, scaled to the rms of the intact stack, $\mathrm{dE}(\mathrm{j}) / \mathrm{dE}$, as a function of $\mathrm{j}$. 